\title{
Multivitamins, Nutrition, and Antiretroviral Therapy for HIV Disease in Africa
}

\author{
Barbara Marston, M.D., and Kevin M. De Cock, M.D.
}

Readers could be forgiven for being confused by the literature on micronutrients and the pathogenesis of human immunodeficiency virus (HIV) disease. Micronutrients play important roles in maintaining immune function and neutralizing the reactive oxygen intermediates produced by activated macrophages and neutrophils in their response to microorganisms. Serum and plasma measurements of vitamins and trace elements, which are imperfect indicators of body stores, have shown that deficiencies are common among HIVinfected persons, especially those who are underprivileged, such as women in developing countries, and injection-drug users. A vicious cycle has been envisaged in which undernourished HIVinfected persons have micronutrient deficiencies, leading to further immunosuppression and oxidative stress and subsequent acceleration of HIV replication and $\mathrm{CD} 4+\mathrm{T}$-cell depletion. ${ }^{1}$

It has been difficult, however, to show causal associations between specific deficiencies and adverse clinical outcomes in HIV-infected persons, and data linking replacement or supplementation of specific micronutrients with a clinical benefit have been sparse and inconsistent. In this issue of the Journal, Fawzi and colleagues report the results of a controlled trial of multivitamin supplementation among HIV-infected pregnant women in Tanzania. ${ }^{2}$ Their report prompts important questions about the plausibility of the findings but also raises broader issues concerning the role of nutrition in the public health response to HIV infection and AIDS in Africa in this era of increasing access to antiretroviral therapy.

The authors compared supplementation consisting of multivitamins alone, vitamin A alone, or both with placebo. They found that women who were randomly assigned to receive multivitamin supplementation were less likely to have progression to advanced stages of HIV disease, had better preservation of CD4+ T-cell counts and lower viral loads, and had lower HIV-related morbidity and mortality rates than women who received placebo. Vitamin A appeared to reduce the effect of multivitamins and, when given alone, had some negative effects.

The credibility of these data is supported by sound study design and methods, appropriate analyses, and compatibility with the limited exist- ing data, such as those showing decreased mortality among HIV-infected Thai adults who received multivitamins. ${ }^{3}$ Fawzi and colleagues have previously shown that multivitamin use is associated with improved pregnancy outcomes, including reduced rates of low birth weight, prematurity, and fetal death. ${ }^{4}$ Although some observational data have associated vitamin A deficiency with increased risks of perinatal transmission and other adverse outcomes, ${ }^{5}$ vitamin A supplementation has reportedly been followed by increased rates of mother-tochild transmission of infection. ${ }^{6}$ On the basis of these previous data as well as the current findings, there seems to be no justification for pursuing vitamin A supplementation as an intervention for HIV infection.

There has been little prior experience with extensive use of multivitamins in African populations, and long-term multivitamin administration has not been considered sustainable. Moreover, there are no solid data regarding the value of multivitamins among HIV-infected adults in the industrialized world, although routine supplementation is often practiced. Thus, before definitive recommendations can be made about routine use of multivitamins in the treatment of persons with HIV infection and AIDS, there is a clear need to confirm the new findings and to evaluate the effects of multivitamins in larger populations, particularly among persons with more advanced HIV disease or more serious nutritional deficiencies.

As a consequence of the recent substantial increase in funding and support for HIV and AIDS treatment programs, the treatment options for HIV disease and AIDS in Africa have changed fundamentally since the study under discussion was initiated. Needing to interpret their results against this altered landscape, Fawzi and colleagues speculate that by reducing the progression of HIV disease, the use of multivitamins may delay the need to institute antiretroviral-drug therapy, thus saving resources as well as preserving therapeutic options.

Individual, small clinical trials rarely form an adequate basis for policy formulation, and they are unlikely to answer convincingly the important questions now raised - specifically, whether multivitamin supplementation can reduce mortality in the context of increased access to antiretroviral- 
drug therapy and whether the initiation of such therapy can be delayed. These critical questions could potentially be answered in Africa by a socalled large, simple trial. ${ }^{7}$ Involving very large populations and assessing only hard end points such as mortality, large, simple trials are especially suitable for evaluating simple treatments that probably have moderate efficacy for common diseases. At a population level, such treatments can have a considerable effect on public health. Without such a study, it is unlikely that adequate evidence will become available to provide scientifically based recommendations concerning the use of multivitamin supplementation for patients with HIV disease and AIDS and for treatment programs in Africa.

Although definitive policy recommendations concerning multivitamin supplementation must await the appropriate evidence (ideally from a large, simple trial in a population with access to antiretroviral drugs), individual treatment programs and clinicians would be justified in routinely prescribing this nutritional support, since it may provide a benefit and does no harm. Multivitamins offer safe opportunities for patients to become accustomed to taking regular medication before beginning antiretroviral therapy. Multivitamins could be offered through home-based care programs that until now have too often ignored even basic biomedical interventions such as HIV testing, the need to rule out tuberculosis, and the provision of trimethoprim-sulfamethoxazole to HIV-infected persons.

Fawzi and colleagues' study of multivitamin supplementation should stimulate broader discussion of the role of nutrition in patients with AIDS in the developing world. With its effects on morbidity, mortality, and poverty, the epidemic of HIV infection and AIDS has worsened food security in Africa, especially in the southern part of the continent, the area most affected by HIV. HIV disease increases metabolic requirements, suppresses appetite, may impair swallowing by causing oral and esophageal opportunistic infections, may be associated with malabsorption due to various gastroenteropathies, and perhaps most important, results in progressive disability and impoverishment, with a consequent inability to raise or afford food. Undernutrition also interacts with HIV in a variety of ways. Undernutrition may promote HIV transmission, for example, when sex is exchanged for money to buy food for oneself and one's family. The clinical picture of the HIV wasting syndrome (also known as the "slim disease"), a characteristic manifestation of AIDS in
Africa, results in large part from undernutrition in the face of opportunistic illnesses, especially tuberculosis, which itself may be made more likely by inadequate food intake. ${ }^{8,9}$ Whatever its cause, a reduced body-mass index is a strong predictor of death.

As donor-funded initiatives expand in Africa, it has become clear that nutrition will have to be addressed in the treatment of HIV disease and AIDS. In the focus-group discussions that we conducted when starting an antiretroviral treatment program in a large Nairobi slum, every group interviewed listed the lack of food as the most likely cause of nonadherence to antiretroviral (ARV) drug therapy. One participant succinctly stated, "If you give us ARVs, please give us food, just food." There truly is irony, not captured in the language of treatment advocacy, in providing antiretroviral drugs to populations that lack access to safe water or food.

The possibility that a relatively inexpensive intervention such as multivitamin supplementation could reduce the risk of or delay HIV-associated disease and death raises the question of how other basic interventions need to be integrated into our nascent HIV and AIDS treatment programs. Africa's health needs extend beyond the treatment of HIV disease and AIDS; HIV-infected persons are at great risk from other threats, as well. For example, programs to prevent mother-to-child transmission of HIV will not fulfill their potential if ineffective maternal and child health services fail to prevent the many competing causes of death in women and children.

An obvious approach is the promotion of simple but important public health interventions alongside and as part of HIV and AIDS treatment initiatives. One example is malaria prevention through the provision of insecticide-impregnated bed nets. In western Kenya's Nyanza Province, an area where the prevalence of antenatal HIV infection is approximately 25 percent, the provision of insecticidetreated nets reduced infant mortality by up to 25 percent at a time when no program existed to prevent mother-to-child transmission of HIV. ${ }^{10}$ Another example is the promotion of point-of-use chlorination to provide safe drinking water; implementation of this system reduced the incidence of diarrhea among HIV-infected adults in Tororo, Uganda, by 30 percent. ${ }^{11}$

Innovative schemes will have to be developed to address the need for food supplementation within HIV and AIDS treatment programs. One such scheme exists near Eldoret, in western Kenya, 
where a farm has been developed on donated land and is tended by some of the patients who receive care at the local clinic. In addition to antiretroviral drugs, food from the farm can be "prescribed" by health care providers for HIV-infected patients and their families.

A final word of caution is required with respect to the implementation of clinical and public health measures in Africa. A fine balance is required between the search for "simple" and "appropriate" interventions and efforts to institute the effective measures that we in the industrialized world take for granted. "Cost-effective" should not be a polite term for cheap, nor should "simple" mean not very effective. As attractive and important as simple interventions are and as massive as the shortage of basic public health infrastructure is, the need for antiretroviral therapy in Africa is real and compelling. The international community must continue to expand its efforts to meet this need.

The opinions and statements expressed in this article are those of the authors and do not represent the official policy, endorsement, or views of the Centers for Disease Control and Prevention, the U.S. Public Health Service, or the Department of Health and Human Services.

From the Centers for Disease Control and Prevention, Nairobi Kenya.
1. Semba RD, Tang AM. Micronutrients and the pathogenesis of human immunodeficiency virus infection. Br J Nutr 1999;81:181-9. 2. Fawzi WW, Msamanga GI, Spiegelman D, et al. A randomized trial of multivitamin supplements and HIV disease progression and mortality. N Engl J Med 2004;351:23-32.

3. Jiamton S, Pepin J, Suttent R, et al. A randomized trial of the impact of multiple micronutrient supplementation on mortality among HIV-infected individuals living in Bangkok. AIDS 2003;17: 2461-9.

4. Fawzi WW, Msamanga GI, Spiegelman D, et al. Randomised trial of effects of vitamin supplements on pregnancy outcomes and T cell counts in HIV-1-infected women in Tanzania. Lancet 1998; 351:1477-82.

5. Semba RD, Miotti PG, Chiphangwi JD, et al. Maternal vitamin A deficiency and mother-to-child transmission of HIV-1. Lancet 1994; 343:1593-7.

6. Fawzi WW, Msamanga GI, Hunter D, et al. Randomized trial of vitamin supplements in relation to transmission of HIV-1 through breastfeeding and early child mortality. AIDS 2002;16:1935-44.

7. Yusuf S, Collins R, Peto R. Why do we need some large, simple randomized trials? Stat Med 1984;3:409-22.

8. Boelaert JR, Gordeuk VR. Protein energy malnutrition and risk of tuberculosis infection. Lancet 2002;360:1102.

9. Lucas SB, De Cock KM, Hounnou A, et al. Contribution of tuberculosis to slim disease in Africa. BMJ 1994;308:1531-3.

10. Phillips-Howard PA, Nahlen BL, Kolczak MS, et al. Efficacy of permethrin-treated bed nets in the prevention of mortality in young children in an area of high perennial malaria transmission in western Kenya. Am J Trop Med Hyg 2003;68:Suppl:23-9.

11. Lule JR, Mermin J, Malamba S. Effect of safe water and cotrimazole on diarrhea among people with HIV. Presented at the 13th International Conference on AIDS and STIs in Africa, Nairobi, Kenya, September 21-26, 2003. abstract.

Copyright (c) 2004 Massachusetts Medical Society. 